\title{
Design and Realization of Memory-Based Chirp Generator on Synthetic Aperture Radar (SAR)
}

\author{
Bagas D. Putera, Erfansyah Ali*, Heroe Wijanto, M. Ramdhani \\ School of Electrical and Engineering, Telkom University \\ *erfansyahali@telkomuniversity.ac.id \\ Manuscript received November 19, 2017; revised April 16, 2018; accepted May 16, 2018
}

\begin{abstract}
Synthetic Aperture Radar (SAR) is a radar system that uses platform movement combined with signal processing. Eventhough it use a small antenna, SAR produce high resolution as good as a large antenna. In SAR imaging, radar is mounted on a moving platform. It transmits electromagnetic pulses and receives backscattered echo signals. Transmitted pulses are subsequently scattered by earth surface and only small portion of them are received by antenna. SAR transmitted signals normally is a chirp or linear frequency modulation (LFM) signal. Chirp signal can be generated by using the analog and digital generator. Digital chirp generator is divided into two methods which are the memory-based and direct digital synthesizer (DDS). The difference of these two methods is located in the memory ROM. In this study, we designed and realized digital chirp generator by using Field Programmable Gate Array (FPGA) DE-1 development board for SAR implementation. It operates in 1.27 GHz (L Band) frequency and had a bandwith of $10 \mathrm{MHz}$ with $24 \mathrm{MHz}$ sampling rate. We found that the output of FPGA is well performed for chirp signal in digital domain.
\end{abstract}

Keywords: Digital chirp generator; SAR; Memory-based chirp generator; FPGA;

DOI: https://doi.org/10.25124/jmecs.v4i1.1695

\section{Introduction}

SAR is a radar system that can obtain images with very high resolution by considering its movement with further signal processing. It create a very big 'synthetic aperture' $\mathrm{N}$ arrays antenna along its movement resulting a very sharp beamwidth. SAR has proved very useful not only in military surveillance but also for topological mapping and snow monitoring with high resolution. Furthermore, it can operate day and night [1].

SAR implements LFM so that high resolution can be achieved with normal pulse width. LFM or chirp signal can be generated either in analog or digital. Digital chirp generators have advantages such as stability, flexibility, and low cost. It can be change or modified easily by changing the firmware and memory contents of the digital generator [2]. In 2015, analog chirp generator has been designed and realized by Nur Aeni [3] and at same year digital chirp is simulated by Meidwita [4]. In this study we designed $10 \mathrm{MHz}$ bandwidth digital chirp, implemented it in FPGA and converted it to analog by using digital to analog converter (DAC).

\section{Design and Implementation}

\subsection{Digital Chirp}

Chirp signal or compressed pulse is a radar pulse with increased or decreased frequency. When the frequency is increased it is called as up-chirp and when the frequency is decreased it is called as down-chirp. The chirp signal equation is expressed in Eq.(1).

$$
x(t)=\operatorname{rect}\left(\frac{t}{T}\right) A e^{j \pi \beta t^{2}}
$$

where the function of $\operatorname{rect}(x)$ and chirp rate is define in Eq.(2) and Eq.(3) respectively.

$$
\begin{gathered}
\operatorname{rect}(x)= \begin{cases}1, & |x|<\frac{1}{2} ; \\
0, & |x|>\frac{1}{2} .\end{cases} \\
\beta= \pm \frac{B}{T}
\end{gathered}
$$

The positive and negative signs in Eq.(3) indicate up-chirp and down-chirp, respectively. The amplitude modulation is $A$ and the phase of LFM signal is $e^{j \pi \beta t^{2}}$ in radians [5] as explained in Eq.(4).

$$
\Phi(t)=\pi \beta t^{2}
$$

The instantaneous frequency $f(t)$ is defined as the derivative of LFM phase as explained in Eq.(5).

$$
f(t)=\frac{1}{2 \pi} \frac{d \Phi(t)}{d t}=\frac{1}{2 \pi} \frac{d\left(\pi \beta t^{2}\right)}{d t}
$$

In SAR systems, chirp signals are typicaly modulated over a frequency badwidth range. A sinusoidal signal is generated when the LFM chirp signal is auto-correlated. 
In digital chirp generator, the shape of the signal can be configured easily by changing the firmware and the memory content. It is realized by using FPGA which consists of counter IC, memory IC, controller, and DAC. FPGAs is using the Hardware Domain Language (HDL) programming language. In FPGA there is a component called Logic Block which can be programmed according to the will of the user [6]. Generally there are two types of digital chirp generator, which are memory-based and DDS. Memory-based chirp generator stores chirp signal form in memory and repeat it. The repeated signal is a preset signal, therefore the accuracy of the signal is relatively higher than DDS. However it needs to be re-configured for other chirp shapes [7].

\subsection{Memory-based Chirp Generator Design}

LFM signal is simulated using Matlab as analog signal therefore it need to be converted into digital format. The digital data will be stored in the memory which will be a fidelity on the FPGA [6]. The block diagram of the memory-based chirp generator is shown by Fig.1.

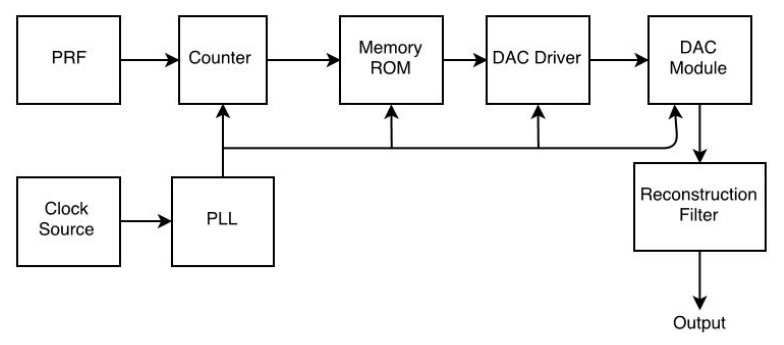

Fig. 1: Memory-based chirp generator block diagram

Binary Counter is a sequential logic which count sequentially from 0 to certain number or until it is ordered to stop. The output of the Binary Counter will be the address of ROM and the data in ROM will be converted to analog signal by using DAC. In this study a digital chirp generator is designed with parameters are shown in Table 1.

Table 1: Chirp design specifications

\begin{tabular}{|l|r|}
\hline Parameter & Value \\
\hline sampling frequency & $24 \mathrm{MHz}$ \\
chirp duration & $5 \mu \mathrm{s}$ \\
min frequency & $0 \mathrm{MHz}$ \\
max frequency & $10 \mathrm{MHz}$ \\
chirp bandwidth & $10 \mathrm{MHz}$ \\
amplitude & $\pm 5 \mathrm{~V}$ \\
quantization & $8 \mathrm{bit}$ \\
\hline
\end{tabular}

At first, chirp signals is simulated in Matlab to get the desired output signal as shown in Eq.(6).

$$
x(t)=A \cos \left(2 \pi f_{t} t\right)
$$

where

$$
\begin{gathered}
f_{t}=k t+f_{0} \\
k=\frac{f_{t}-f_{0}}{T}
\end{gathered}
$$

$A, k, f_{0}, f_{t}$ and $T$ is chirp amplitude, chirp rate, start frequency, end frequency and chirp duration respectively. The equation resulting chirp signal form as shon in Fig.2. The chirp designed with unsigned value therefore the amplitude is $0-2 \mathrm{~V}$ eventhough in Table 1 stated that it is $\pm 5 \mathrm{~V}$. However the chirp duration is $5 \mu \mathrm{s}$ as specification and it is represented in 1000 samples.

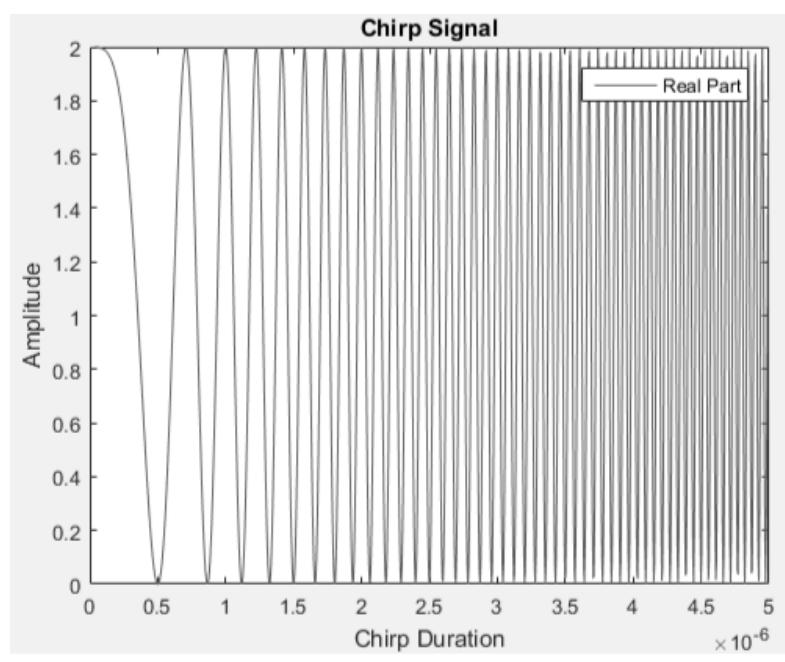

Fig. 2: Chirp signal simulated in Matlab

\subsection{FPGA Implementation}

Before the output data was implemented in FPGA, it was need to be pre-processed in Altera Quartus II by using VHDL programming language. The output data is in real number and need to be converted to 8 bits binary therefore it can be read in FPGA. The output data was transform to pulses as shown in Fig.3, however for better image resolution it has been cropped to single pulse.

Binary Counter counted from 0-999 to represented 1000 samples and looping. In FPGA implementation, it started with $50 \mathrm{MHz}$ input clock and end at 8 pins GPIO which would connected to 8 bits DAC module. We used Altera DE-1 Development and Education Board [8] which detail specification is shown in Table 2.

\subsection{DAC Module}

By using DAC0808 IC[9], the FPGA output data can be displayed in the osciloscope. It converted 8bits digital data to analog current. It was coupled by TL081 Op-Amp [10] in order to convert current into voltage. The design and realization of DAC module used in this study is shown in Fig.4. Fig.5 shows when it was connected to FPGA board. 
Putera et al / Journal of Measurements, Electronics, Communications, and Systems


Fig. 3: Chirp signal simulated in Quartus

Table 2: Altera DE-1 specifications

\begin{tabular}{|l|l|}
\hline Hardware & Specifications \\
\hline FPGA & Altera Cyclone II 2C20 \\
\hline Memory & $\begin{array}{l}512 \mathrm{~KB}(256 \mathrm{~K} \times 16) \text { SRAM } \\
4 \mathrm{MB} \text { Flash }\end{array}$ \\
\hline Clock & $\begin{array}{l}50 \mathrm{MHz}, 24 \mathrm{MHz}, 27 \mathrm{MHz}, \\
\text { or external clock sources }\end{array}$ \\
\hline I/O & Two 40-pin Expansion Headers \\
\hline Display & 10 red and 8 green LED \\
\hline
\end{tabular}
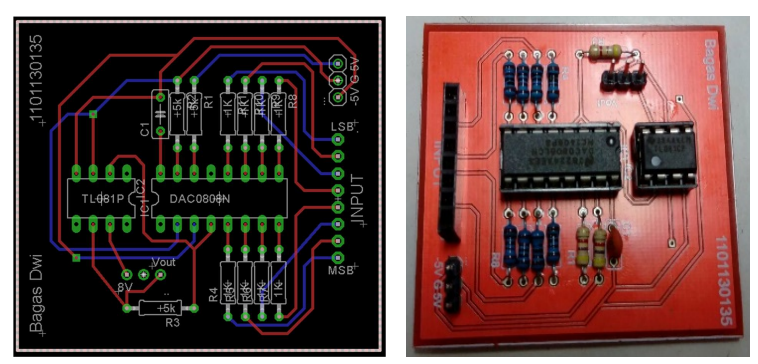

Fig. 4: DAC module

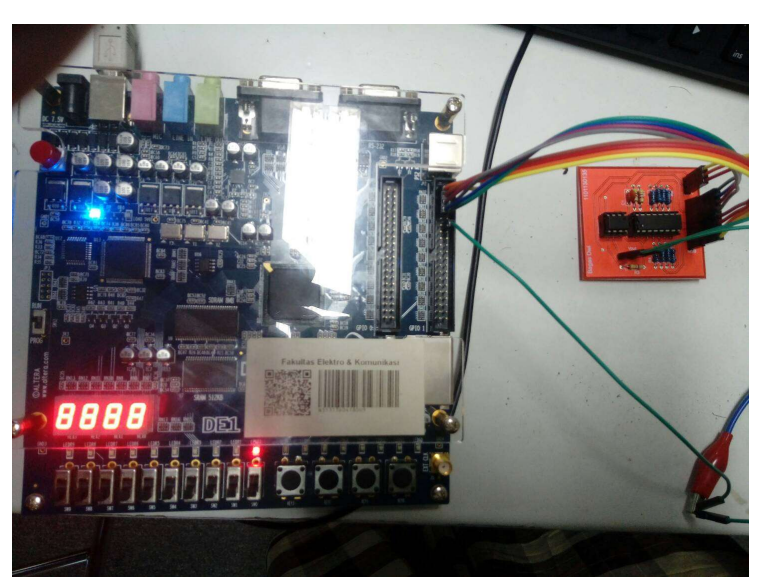

Fig. 5: Digital chirp module

\section{Result and Analysis}

Parameter measurements on each component of the compiler generator chirp was done to compare between system designed and the realization. The measured components include the DAC module and the result of chirp output signal.

\subsection{DAC Module}

A comparison between design and realization of DAC module is shown at Table 3. It was measured to obtain the accuracy level. The DAC module have accuracy $85 \%$ which maximum error less than $0.03 \mathrm{~V}$. The IC TL081 need to be powered by $8 \mathrm{~V}$ instead of $5 \mathrm{~V}$ at $V^{+}$pin to obtain $5 \mathrm{~V}$ at 11111111 digital input.

Table 3: DAC module comparison

\begin{tabular}{|c|c|c|}
\hline Input A1-A8 & $V_{o}$ Design & $V_{o}$ Realization \\
\hline 00000000 & $0 \mathrm{~V}$ & $0 \mathrm{~V}$ \\
00000001 & $19.53 \mathrm{mV}$ & $16.9 \mathrm{mV}$ \\
00000011 & $58.59 \mathrm{mV}$ & $55.5 \mathrm{mV}$ \\
00000111 & $136.71 \mathrm{mV}$ & $134.1 \mathrm{mV}$ \\
00001111 & $292.96 \mathrm{mV}$ & $291.4 \mathrm{mV}$ \\
00011111 & $605.46 \mathrm{mV}$ & $602.2 \mathrm{mV}$ \\
00111111 & $1.23 \mathrm{~V}$ & $1.23 \mathrm{~V}$ \\
01111111 & $2.48 \mathrm{~V}$ & $2.496 \mathrm{~V}$ \\
10000000 & $2.5 \mathrm{~V}$ & $2.519 \mathrm{~V}$ \\
10000001 & $2.519 \mathrm{~V}$ & $2.538 \mathrm{~V}$ \\
10000011 & $2.558 \mathrm{~V}$ & $2.576 \mathrm{~V}$ \\
10000111 & $2.636 \mathrm{~V}$ & $2.655 \mathrm{~V}$ \\
10001111 & $2.792 \mathrm{~V}$ & $2.812 \mathrm{~V}$ \\
10011111 & $3.1 \mathrm{~V}$ & $3.125 \mathrm{~V}$ \\
10111111 & $3.73 \mathrm{~V}$ & $3.753 \mathrm{~V}$ \\
11111111 & $4.98 \mathrm{~V}$ & $5.02 \mathrm{~V}$ \\
\hline
\end{tabular}

\subsection{Chirp Signal Verification}

The output from DAC module subsequently was connected to oscilloscope resulting analog chirp as shown in Fig.6. The comparison between design and realization is shown at Table.4. The amplitude realization is change to $0-5 \mathrm{~V}$ instead of $\pm 5 \mathrm{~V}$ because of DAC0808 can not read signed bits. The sampling frequency is downsized to $6 \mathrm{MHz}$ because of DAC0808 settling time is $150 \mathrm{~ns}$ or maximum frequency sampling supported is $6.67 \mathrm{MHz}$.

Table 4: Comparison between design and realization

\begin{tabular}{|l|c|c|}
\hline Parameter & Design & Realization \\
\hline f. sampling & $24 \mathrm{MHz}$ & $6 \mathrm{MHz}$ \\
f. range & $0-10 \mathrm{MHz}$ & $0-10 \mathrm{MHz}$ \\
amplitude & $\pm 5 \mathrm{~V}$ & $0-5 \mathrm{~V}$ \\
quantization & $8 \mathrm{bit}$ & $8 \mathrm{bit}$ \\
\hline
\end{tabular}

\section{Conclusion}

From the whole process of design, realization, and measurement of chirp digital generator, it can be concluded that digital chirp generator work as designed with several limitations caused by DAC0808. 
Putera et al / Journal of Measurements, Electronics, Communications, and Systems

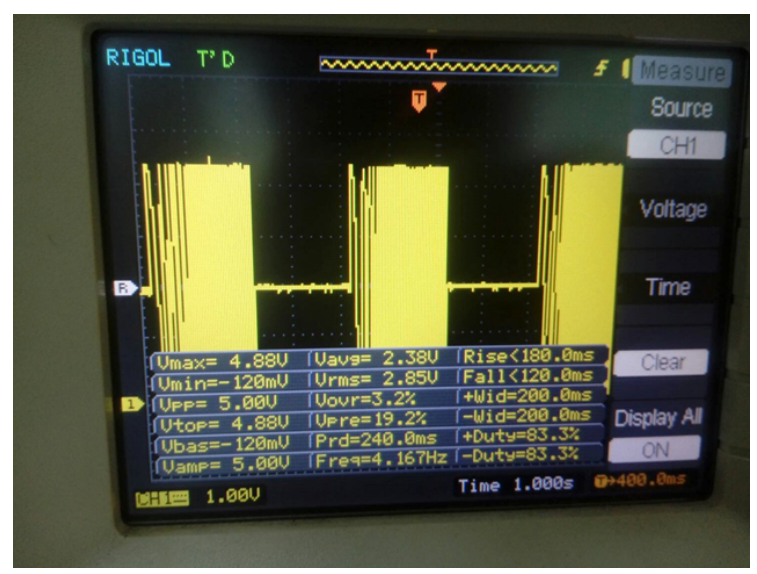

Fig. 6: Digital chirp displayed at oscilloscope

Its frequency sampling limitations and the unability to read signed bits resulting a downgrade in realization of digital chirp. In the future the system need to implement higher specification DAC chip, because Altera Cyclone II FPGA can support output clock up to $50 \mathrm{MHz}$.

\section{References}

[1] A. Moreira, "Synthetic Aperture Radar (SAR): Principles and applications," German Aerospace Center, 2013.

[2] A. Moreira, P. Prats-Iraola, M. Younis, G. Krieger, I. Hajnsek, and K. Papathanassiou, "A Tutorial on Synthetic Aperture Radar," IEEE Geoscience and Remote Sensing Magazine (GRSM), vol. 1, pp. 6-43, 032013.

[3] N. Aeni, Perancangan dan Realisasi Generator Chirp Pada Synthetic Aperture Radar (SAR). Telkom University, 2015.

[4] Meidwita, Analisis dan Simulasi Pengolahan Sinyal Chirp Pada Sinyal Synthetic Aperture Radar (SAR). Telkom University, 2015.

[5] L. Nicolaescu and T. Oroian, "Radar Cross Section," in 5th International Conference on Telecommunications in Modern Satellite, Cable and Broadcasting Service. TELSIKS 2001. Proceedings of Papers (Cat. No.01EX517), vol. 1, pp. 65-68 vol.1, Sept 2001.

[6] M. Y. Chua and V. Chet Koo, "FPGA-based Chirp Generator for High Resolution UAV SAR," Progress in Electromagnetics Research-pier - PROG ELECTROMAGN RES, vol. 99, pp. 7188, 012009.

[7] H. Yang, S.-B. Ryu, H.-C. Lee, S.-G. Lee, S.-S. Yong, and J.-H. Kim, "Implementation of DDS Chirp Signal Generator on FPGA," pp. 956-959, 102014

[8] Altera DE-1 Development and Education Board, User Manual.

[9] Texas Instrument, DAC0808 datasheet, 1999.

[10] Texas Instrument, TL081 datasheet, 2015. 